\title{
dc and ac Josephson effect in a superconductor-Luttinger-liquid-superconductor system
}

\author{
Rosario Fazio \\ Institut für Theoretische Festkörperphysik, Universtität Karlsruhe, 76128 Karlsruhe, Germany \\ and Istituto di Fisica, Università di Catania, viale A. Doria 6, 95129 Catania, Italy* \\ F. W. J. Hekking \\ Theoretical Physics Institute, University of Minnesota, 116 Church Street SE, Minneapolis, Minnesota 55455 \\ A. A. Odintsov \\ Department of Applied Physics, Delft University of Technology, P.O. Box 5046, 2600 GA Delft, The Netherlands \\ and Nuclear Physics Institute, Moscow State University, Moscow 119899 GSP, Russia*
}

(Received 21 September 1995)

\begin{abstract}
We calculate both the dc and the ac Josephson current through a one-dimensional system of interacting electrons, connected to two superconductors by tunnel junctions. We treat the (repulsive) Coulomb interaction in the framework of the one-channel, spin-1/2 Luttinger model. The Josephson current is obtained for two geometries of experimental relevance: a quantum wire and a ring. At zero temperature, the critical current is found to decay algebraically with increasing distance $d$ between the junctions. The decay is characterized by an exponent which depends on the strength of the interaction. At finite temperatures $T$, lower than the superconducting transition temperature $T_{c}$, there is a crossover from algebraic to exponential decay of the critical current as a function of $d$, at a distance of the order of $\hbar v_{F} / k_{B} T$. Moreover, the dependence of critical current on temperature shows nonmonotonic behavior. If the Luttinger liquid is confined to a ring of circumference $L$, coupled capacitively to a gate voltage and threaded by a magnetic flux, the Josephson current shows remarkable parity effects under the variation of these parameters. For some values of the gate voltage and applied flux, the ring acts as a $\pi$ junction. These features are robust against thermal fluctuations up to temperatures on the order of $\hbar v_{F} / k_{B} L$. For the wire geometry, we have also studied the ac-Josephson effect. The amplitude and the phase of the time-dependent Josephson current are affected by electron-electron interactions. Specifically, the amplitude shows pronounced oscillations as a function of the bias voltage due to the difference between the velocities of spin and charge excitations in the Luttinger liquid. Therefore, the acJosephson effect can be used as a tool for the observation of spin-charge separation.
\end{abstract}

\section{INTRODUCTION}

Due to the recent development of superconductorsemiconductor $(\mathrm{S}-\mathrm{Sc})$ integration technology it has become possible to observe the transport of Cooper pairs through $\mathrm{S}-\mathrm{Sc}$ mesoscopic interfaces. Examples are the supercurrent through a two-dimensional electron gas (2DEG) with $\mathrm{Nb}$ contacts ( $\mathrm{S}-\mathrm{Sc}-\mathrm{S}$ junction $)^{1}$ and excess low-voltage conductance due to Andreev scattering in Nb-InGaAs (S-Sc) junctions. $^{2}$ The transfer of single electrons through the interface between a semiconductor and a superconductor with energy gap $\Delta$ is exponentially suppressed at low temperatures and bias voltages $k_{B} T, e V \ll \Delta$ ( $e$ is the electron charge). Instead, electrons will be transferred in pairs through the interface, a phenomenon known as Andreev reflection. ${ }^{3}$ It has been realized only recently, that the phase coherence between the two electrons involved in this process could give rise to distinct signatures in the transport properties of mesoscopic $\mathrm{S}-\mathrm{Sc}-\mathrm{S}$ and $\mathrm{S}-\mathrm{Sc}$ systems. ${ }^{4,5}$

If the normal $(\mathrm{Sc})$ region is free of disorder, the propagation of electrons is ballistic. Phase coherence between the two electrons is maintained over the length $L_{\text {cor }}=\hbar v_{F} / \max \left\{k_{B} T, e V\right\}$, where $v_{F}$ is the Fermi velocity. In this regime, the critical current $I_{c}$ through a short and narrow constriction in a high-mobility noninteracting 2DEG, connected to two superconductors should be quantized: ${ }^{6}$ each propagating mode contributes an amount $e \Delta / \hbar$ to the critical current.

In the presence of disorder in the normal region, the motion of the two electrons will be diffusive. Like in disordered metals, the phase coherence between the two electrons is limited by the correlation length $L_{\text {cor }}=\sqrt{\hbar D / \max \left\{k_{B} T, e V\right\}}$, where $D$ is the diffusion constant. For instance, the excess low-voltage conductance in $\mathrm{S}-\mathrm{Sc}$ junctions ${ }^{2}$ can be explained in terms of constructive interference occurring over this length scale between the two electrons incident on the $\mathrm{S}-\mathrm{Sc}$ interface. ${ }^{7,8}$

In these examples, electron-electron interactions are neglected. It is well known, though, that they may have a strong influence on the transport properties of mesoscopic systems. In general the interactions modify the phasecoherence length $L_{\text {cor }}$, which poses limitations on the abovementioned mesoscopic effects. In specific cases the effects of electron-electron interactions will strongly depend on the layout of the system under consideration.

For example, the interactions will modify the critical current $I_{c}$ through a normal metallic slab sandwiched between two superconductors. ${ }^{9}$ If the coupling between normal metal and superconductors is weak (tunneling regime) and the size of the slab (and hence its electric capacitance $C$ ) is small, a 
phenomenological capacitive model ${ }^{10}$ can be used to describe the effect of interactions. As a result the critical current shows strong resonant dependence on the electrochemical potential of the slab dependence has different character for $E_{C}<\Delta$ and $E_{C}>\Delta, E_{C}=e^{2} / 2 C$ being the charging energy. On the other hand, if the normal metal and the superconductor are well coupled (regime of Andreev reflection), electron-electron interactions will modify the results obtained in Ref. 9 in quite a different fashion. A perturbative treatment of the interactions ${ }^{12}$ shows that an additional supercurrent through the slab arises, whose sign depends on the nature of the interactions in the slab (attractive or repulsive), and whose phase dependence has period $\pi$ (rather than $2 \pi$ in the noninteracting case).

If instead of a metal a low-dimensional Sc nanostructure with a small electron concentration is considered, the abovementioned descriptions of the electron-electron interactions are no longer sufficient. In one-dimensional (1D) systems the Coulomb interaction cannot be treated as a weak perturbation. As a result a nonperturbative, microscopic treatment of interactions is required. For 1D systems this can be done in the framework of the Luttinger model. ${ }^{13}$ Interactions have a drastic consequence: there are no fermionic quasiparticle excitations. Instead, the low-energy excitations of the system consist of independent long-wavelength oscillations of the charge and spin density, which propagate with different velocities. The density of states has power-law asymptotics at low energies and the transport properties cannot be described in terms of the conventional Fermi-liquid approach. For a quantum wire with an arbitrarily small barrier this leads to a complete supression of transport at low energies. ${ }^{14-17}$

Another interesting feature arises in 1D interacting systems of a finite size. For a Luttinger liquid confined to a ring, $\operatorname{Loss}^{18}$ found remarkable parity effects ${ }^{19}$ for the persistent currents. He used the concept of Haldane's topological excitations, ${ }^{20}$ extending the previous work of Byers and Yang for noninteracting electrons in a ring. ${ }^{21}$ Depending on the parity of the total number of electrons on the ring, the ground state is either diamagnetic or paramagnetic. For spin-1/2 electrons an additional sensitivity on the electron number modulo 4 has been found. ${ }^{22}$ Experimental evidence for Luttinger-liquid behavior in Sc nanostructures has been found recently. The dispersion of separate spin and charge excitations in GaAs/AlGaAs quantum wires has been measured with resonant inelastic light scattering. ${ }^{23}$ Transport measurements on quantum wires have revealed power-law dependence of the conductance as a function of temperature. ${ }^{24}$

In view of this we expect that electron-electron interactions may well have drastic, observable consequences in systems which consist of low-dimensional Sc nanostructures connected to superconductors. In this paper, we will study the Josephson current through a Luttinger liquid. ${ }^{25,26}$ Specifically, we consider two geometries which can be realized experimentally: a long wire with contacts to two superconductors at a distance $d$ [see Fig. 1(a)] or a ring-shaped Luttinger liquid shown in Fig. 1(b). In both cases the 1D electron liquid is connected to the superconducting electrodes by tunnel junctions. This is an interesting system from various points of view. First of all, it enables one to study in a microscopic way how the Coulomb interaction influences the

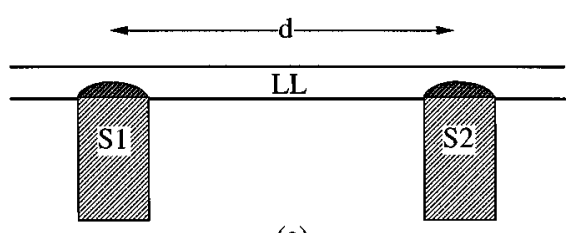

(a)

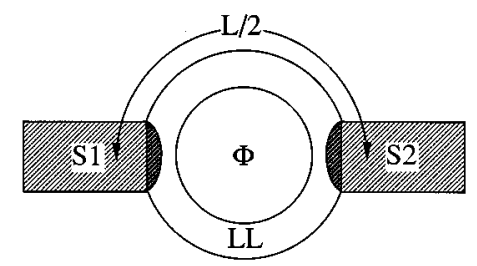

(b)

FIG. 1. The geometries discussed in the text: (a) onedimensional wire connected to two superconductors by tunnel junctions. The distance between the junctions is $d$. (b) Ring with circumference $L$ connected to two superconductors by tunnel junctions. The distance between the junctions is $L / 2$, the ring is threaded by a magnetic flux $\Phi$.

phase-coherent propagation of two electrons through a 1D normal region. ${ }^{27}$ Secondly, various aspects of transport in mesoscopic systems (parity effects and interference combined with electron-electron interactions) and their interplay can be enlightened in such a device. Finally, since the Josephson effect is a ground-state property, the Josephson current can be used as a tool to probe the ground state of an interacting electron system. In particular, for the ring geometry in the presence of an Aharonov-Bohm flux, the various possible ground-state configurations can be determined by studying flux and gate voltage dependence of the critical current.

The paper is organized as follows. In Sec. II we briefly review the properties of the spin-1/2 Luttinger model. In Sec. III the general formalism for the dc-Josephson effect is presented. The dc-Josephson current is obtained by evaluating the contribution to the free energy which depends on the difference of the superconducting phases. Starting from the general expression (12) we then consider various interesting limiting situations. In Sec. IV, the wire geometry is considered [see Fig. 1(a)]. The critical current decays as a power of the distance $d$ between the contacts. The exponent depends on the interaction strength. We distinguish two cases in which the characteristic energy $\hbar v_{F} / d$ for the $1 \mathrm{D}$ system is either much smaller or much larger than the superconducting gap $\Delta$. For the ring geometry [Sec. V, see Fig. 1(b)], we focus on the dependence of the critical current on the applied gate voltage and/or flux. Both Secs. IV and V contain a discussion of the effect of finite temperatures. Section VI is devoted to the ac-Josephson effect. In this case the imaginary time approach of the previous sections is inadequate and we will use a real-time formulation. The amplitude of the ac component is found to show oscillations as a function of voltage due to spin-charge separation. In the last section we present the conclusions.

\section{THE SPIN-1/2 LUTTINGER LIQUID}

We start the description of the model we use by reviewing the theory of $1 \mathrm{D}$ interacting spin-1/2 fermions (throughout, 
we use $\hbar=k_{B}=1$ ). The long-wavelength behavior of such a quantum wire of length $L$ is governed by the Hamiltonian ${ }^{20}$

$$
\hat{H}_{L}=\int_{-L / 2}^{L / 2} \frac{d x}{\pi} \sum_{j} v_{j}\left[\frac{g_{j}}{2}\left(\nabla \phi_{j}\right)^{2}+\frac{2}{g_{j}}\left(\nabla \theta_{j}\right)^{2}\right] .
$$

It is written as a sum of the contributions from the spin $(j=\sigma)$ and charge $(j=\rho)$ degrees of freedom. The parameters $g_{j}$ denote the interaction strengths and $v_{j}=2 v_{F} / g_{j}$ the velocities of spin and charge excitations. ${ }^{14}$ The parameters $g_{j}$ can be determined once one defines an appropriate microscopic Hamiltonian (e.g., the Hubbard model); an approximate form for the spinless case has been given in Refs. 14 and 15. In this paper we will neglect backscattering (via umklapp or impurity scattering) and restrict ourselves to repulsive, spin-independent interactions. As a result we have $g_{\sigma}=2$ and $v_{\sigma}=v_{F} \cdot{ }^{14}$

We also introduced bosonic fields $\phi_{j}$ and $\theta_{j}$. They are related to the fields $\phi_{s}=\phi_{\rho}+s \phi_{\sigma}$ and $\theta_{s}=\theta_{\rho}+s \theta_{\sigma}$ for spin up $(s=+1)$ and down $(s=-1)$ fermions. These fields obey the commutation relation $\left[\phi_{s}(x), \theta_{s^{\prime}}\left(x^{\prime}\right)\right]$ $=(i \pi / 2) \operatorname{sign}\left(x^{\prime}-x\right) \delta_{s, s^{\prime}}$. The fermionic field operators $\hat{\Psi}$ can be expressed in terms of the spin and charge degrees of freedom: ${ }^{18,20}$

$$
\begin{aligned}
\hat{\Psi}_{L, s}^{\dagger}(x)= & \sqrt{\rho_{0}} \sum_{n, \text { odd }} \exp \left\{\operatorname{ink}_{F} x\right\} \\
& \times \exp \left\{\operatorname{in}\left[\theta_{\rho}+s \theta_{\sigma}\right]\right\} \exp \left\{i\left[\phi_{\rho}+s \phi_{\sigma}\right]\right\},
\end{aligned}
$$

where $k_{F}$ is the Fermi-wave vector and $\rho_{0} \equiv N_{0} / L$ is the average electron density per spin direction. The number $N_{0}$ determines the linearization point of the original electron spectrum, $k_{F} \equiv \pi N_{0} / L .^{18}$

If the wire is closed to form a loop, the periodic condition

$$
\hat{\Psi}_{L, s}(x+L)=\hat{\Psi}_{L, s}(x)
$$

should be imposed on the Fermi operators (2). The fields $\theta$ and $\phi$ can then be decomposed in terms of bosonic fields $\bar{\theta}$ and $\bar{\phi}$ and topological excitations: ${ }^{18,20}$

$$
\begin{gathered}
\theta_{j}(x)=\bar{\theta}_{j}(x)+\theta_{j}^{0}+\pi M_{j}(x / 2 L), \\
\phi_{j}(x)=\bar{\phi}_{j}(x)+\phi_{j}^{0}+\pi J_{j}[(x+L / 2) / 2 L] .
\end{gathered}
$$

Here, $\bar{\theta}_{j}$ and $\bar{\phi}_{j}$ are given by

$$
\begin{gathered}
\bar{\theta}_{j}(x)=\frac{i}{2} \sqrt{\frac{g_{j}}{2}} \sum_{q \neq 0}\left|\frac{\pi}{q L}\right|^{1 / 2} \operatorname{sign}(q) e^{i q x}\left(\hat{b}_{j, q}^{\dagger}+\hat{b}_{j,-q}\right), \\
\bar{\phi}_{j}(x)=\frac{i}{2} \sqrt{\frac{2}{g_{j}}} \sum_{q \neq 0}\left|\frac{\pi}{q L}\right|^{1 / 2} e^{i q x}\left(\hat{b}_{j, q}^{\dagger}-\hat{b}_{j,-q}\right),
\end{gathered}
$$

where $\hat{b}_{j, q}, \hat{b}_{j, q}^{\dagger}$ are Bose operators.

The boundary condition (3) gives rise to the topological excitations $M_{j}$ and $J_{j}$ for the spin and charge degrees of freedom. They are related to the usual topological excitations for fermions with spin $s: M_{s}=(1 / 2)\left[M_{\rho}+s M_{\sigma}\right]$ and $J_{s}=(1 / 2)\left[J_{\rho}+s J_{\sigma}\right]$. Physically, the number $M_{s}$ denotes the number of excess electrons in the Luttinger liquid (LL) with spin $s$ in addition to $N_{0}$. The number $J_{s}$ is the number of current quanta $e v_{F} / L$, carried by electrons with spin $s$. Here, $v_{F}=\pi \rho_{0} / m$ is the Fermi velocity, with $m$ being the electron mass. A net current $J_{\rho} e v_{F} / L$ flows through the quantum wire if there is an imbalance between the number electrons moving to the right and to the left. Using the boundary condition (3) one obtains topological constraints for $M_{s}$ and $J_{s},{ }^{18,20}$ which lead to the following constraints for $M_{j}$ and $J_{j}$ :

(i) The topological numbers $M_{j}$ and $J_{j}$ are either simultaneously even or simultaneously odd;

(ii) when $N_{0}$ is $o d d$ the sum $M_{\rho} \pm M_{\sigma}+J_{\rho} \pm J_{\sigma}$ takes values $\ldots,-4,0,4, \ldots$, when $N_{0}$ is even the sum $M_{\rho} \pm M_{\sigma}+J_{\rho} \pm J_{\sigma}$ takes values $\ldots,-6,-2,2,6, \ldots$.

An Aharonov-Bohm flux $\Phi$ threading the loop couples to the net current, characterized by the topological number $J_{\rho}$ of the field $\phi_{\rho}$. The flux can be incorporated into the Hamiltonian (1) by changing

$$
\nabla \phi_{j} \rightarrow \nabla \phi_{j}-(2 \pi / L) \delta_{j, \rho} f_{\Phi},
$$

where $f_{\Phi}=\Phi / \Phi_{0}$ is the flux frustration and $\Phi_{0}$ is the flux quantum $h / e$.

Since the LL is brought into the contact with particle reservoirs (superconductors) kept at fixed electrochemical potential $\mu$, the number of particles (characterized by the topological number $M_{\rho}$ of the field $\theta_{\rho}$ ) should be coupled to $\mu$. This can be achieved by replacing

$$
\nabla \theta_{j} \rightarrow \nabla \theta_{j}-(2 \pi / L) \delta_{j, \rho} f_{\mu}
$$

in the Hamiltonian (1). The parameter $f_{\mu}=\left(g_{\rho} L / 4 \pi v_{\rho}\right) \Delta \mu$ is related to the difference $\Delta \mu$ between $\mu$ and the Fermi energy $E_{F}=k_{F}^{2} / 2 m$ of the quantum wire, corresponding to the linearization point. Generally, the reference point $\Delta \mu=0$ is defined from the requirement that for $\Phi=0$ there are $2 N_{0}$ electrons in the ground state and the energies to add/remove electrons to/from the system are equal. The difference $\Delta \mu$ can be controlled, e.g., by a gate voltage.

Using Eqs. (1), (4a), (4b), (5a), and (5b) one concludes that the Hamiltonian can be decomposed into nonzero modes and topological excitations:

$$
\begin{aligned}
\hat{H}_{L}= & \sum_{j=\rho, \sigma}\left\{\sum_{q \neq 0} v_{j}|q| \hat{b}_{q, j}^{\dagger} \hat{b}_{q, j}+\frac{\pi v_{j}}{4 L}\left[\frac{g_{j}}{2}\left(J_{j}-4 \delta_{j, \rho} f_{\Phi}\right)^{2}\right.\right. \\
& \left.\left.+\frac{2}{g_{j}}\left(M_{j}-4 \delta_{j, \rho} f_{\mu}\right)^{2}\right]\right\} .
\end{aligned}
$$

Since this Hamiltonian is quadratic in the Bose operators, it is possible to obtain all the correlation functions exactly.

\section{III. dc-JOSEPHSON EFFECT}

Both systems depicted in Fig. 1 can be described by the Hamiltonian

$$
\hat{H}=\hat{H}_{S 1}+\hat{H}_{S 2}+\hat{H}_{L}+\hat{H}_{T} \equiv \hat{H}_{0}+\hat{H}_{T} .
$$

Here, $\hat{H}_{S 1}, \hat{H}_{S 2}$ are the BCS Hamiltonians for the bulk superconductors kept at constant phase difference $\chi=\chi_{S 1}-\chi_{S 2}$. For simplicity we assume equal magnitude 
$\Delta$ for both order parameters. The tunneling between the superconductors and the 1D electron system is described by $\hat{H}_{T}$. It is assumed to occur through two tunnel junctions ${ }^{28}$ at the points $x=0$ and $x=d$,

$$
\begin{aligned}
\hat{H}_{T}= & \sum_{s} T_{1} \hat{\Psi}_{S 1, s}^{\dagger}(x=0) \hat{\Psi}_{L, s}(x=0) \\
& +T_{2} \hat{\Psi}_{S 2, s}^{\dagger}(x=d) \hat{\Psi}_{L, s}(x=d)+(\text { H.c. }) .
\end{aligned}
$$

The constant tunnel matrix elements $T_{1,2}$ can be related to the tunnel conductances $G_{1,2}$ of the junctions, $G_{i}=4 \pi e^{2} N_{L}(0) N_{i}(0)\left|T_{i}\right|^{2}$, where $N_{L}(0)=1 / \pi v_{F}$, and $N_{i}$ is the normal density of states in the superconductors $(i=1,2)$.
The stationary Josephson effect can be obtained by evaluating the phase-dependent part of the free energy $\mathscr{F}(\chi)$. The Josephson current is then given by

$$
I_{J}=-2 e \partial \mathscr{F} / \partial \chi
$$

We expand $\mathscr{F}=-(1 / \beta) \ln Z$, where $Z=\operatorname{Tr} \exp \{-\beta \hat{H}\}$ and $\beta=1 / T$, in powers of the tunneling Hamiltonian $\hat{H}_{T}$, using standard imaginary-time perturbation theory. ${ }^{29}$ The lowest order phase-dependent contribution arises in fourth order. Using Eq. (10) we see that there are 24 contributions to the phase-dependent part of $\mathscr{F}$ :

$$
\begin{aligned}
\mathscr{F}(\chi)= & -\frac{1}{\beta} \int_{0}^{\beta} d \tau_{1} \int_{0}^{\tau_{1}} d \tau_{2} \int_{0}^{\tau_{2}} d \tau_{3} \int_{0}^{\tau_{3}} d \tau_{4}\left\{F_{S 1}^{\dagger}\left(0 ; \tau_{1}-\tau_{2}\right) T_{1}^{2} \Pi_{L}^{(a)}\left(0, d ; \tau_{1}, \ldots, \tau_{4}\right)\left(T_{2}^{*}\right)^{2} F_{S 2}\left(0 ; \tau_{3}-\tau_{4}\right)\right. \\
& \left.+F_{S 1}^{\dagger}\left(0 ; \tau_{1}-\tau_{4}\right) T_{1}^{2} \Pi_{L}^{(b)}\left(0, d ; \tau_{1}, \ldots, \tau_{4}\right)\left(T_{2}^{*}\right)^{2} F_{S 2}\left(0 ; \tau_{2}-\tau_{3}\right)+(\text { H.c. })\right\}+22 \text { similar terms. }
\end{aligned}
$$

This result has a clear physical meaning, see Fig. 2. The Josephson effect consists of processes in which a Cooper pair tunnels from superconductor $S 2$ into the LL with an amplitude $\left(T_{2}^{*}\right)^{2}$. After propagation through the LL, it tunnels into superconductor $S 1$ with an amplitude $\left(T_{1}\right)^{2}$. The Hermitian conjugate terms describe processes in the opposite direction. The propagation in the superconductors is described by the anomalous Green's function

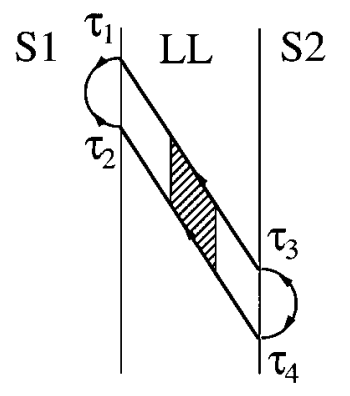

(a)

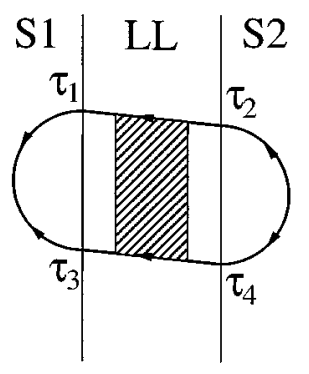

(b)

FIG. 2. Relevant diagram for Josephson tunneling in the limiting cases (a) $v_{F} / d \ll \Delta$ and (b) $v_{F} / d \gg \Delta$. The shaded area indicates the electron-electron interaction.

$$
\begin{aligned}
F_{S i}\left(0 ; \tau-\tau^{\prime}\right) & \equiv\left\langle\mathrm{T}_{\tau} \hat{\Psi}_{S i,-}(d, \tau) \hat{\Psi}_{S i,+}\left(d, \tau^{\prime}\right)\right\rangle_{S i} \\
& =\frac{\pi N(0)}{\beta} \sum_{\omega_{n}} e^{-i \omega_{n}\left(\tau-\tau^{\prime}\right)} \frac{\Delta e^{i \chi_{S i}}}{\sqrt{\omega_{n}^{2}+\Delta^{2}}}
\end{aligned}
$$

where $\langle\cdots\rangle_{S i}$ indicates an average with respect to $\hat{H}_{S i}$. Propagation through the LL is determined by the Cooperon propagator $\Pi_{L}\left(0, d ; \tau_{1}, \ldots, \tau_{4}\right)$. These 24 terms are obtained by considering all possible time-ordered pairs of tunneling events $\tau_{i}, \tau_{j}\left(\tau_{i}<\tau_{j}\right)$ at $x=0$ and $x=d$ together with all possible spin configurations. However, which of these terms are important depends on the relation between the characteristic energy $v_{F} / d$ for the $1 \mathrm{D}$ system and the superconducting gap $\Delta .{ }^{30}$

If the distance between the contacts is large, $v_{F} / d \ll \Delta$, a generic process consists of fast tunneling of two electrons from the superconductor into the 1D system and their slow propagation through the LL. Such a process is illustrated by the first term in Eq. (12). Here,

$$
\begin{aligned}
& \Pi_{L}^{(a)}\left(0, d ; \tau_{1}, \ldots, \tau_{4}\right) \\
& \quad=\left\langle\hat{\Psi}_{L,+}\left(0, \tau_{1}\right) \hat{\Psi}_{L,-}\left(0, \tau_{2}\right) \hat{\Psi}_{L,+}^{\dagger}\left(d, \tau_{3}\right) \hat{\Psi}_{L,-}^{\dagger}\left(d, \tau_{4}\right)\right\rangle
\end{aligned}
$$

where $\left|\tau_{1}-\tau_{3}\right| \sim d / v_{F} \gg\left|\tau_{1}-\tau_{2}\right| \sim\left|\tau_{3}-\tau_{4}\right| \sim 1 / \Delta$ [see Fig. 2(a)]. The average is taken over equilibrium fluctuations in the LL (described by $\hat{H}_{L}$ ). The other relevant processes come from diagrams which are obtained from the one in Fig. 2(a) by means of particle-hole conjugation and by changing the time ordering.

In the opposite limit $v_{F} / d \gg \Delta$, diagrams of the type depicted in Fig. 2(a) are no longer relevant. Instead, one should consider fast and independent propagation of two electrons 
through the LL and slow tunneling between $S$ and LL. This is illustrated by the second term in Eq. (12) [see also Fig. 2(b)], where

$$
\begin{aligned}
& \Pi_{L}^{(b)}\left(0, d ; \tau_{1}, \ldots, \tau_{4}\right) \\
& \quad=\left\langle\hat{\Psi}_{L,+}\left(0, \tau_{1}\right) \hat{\Psi}_{L,+}^{\dagger}\left(d, \tau_{2}\right) \hat{\Psi}_{L,-}\left(0, \tau_{3}\right) \hat{\Psi}_{L,-}^{\dagger}\left(d, \tau_{4}\right)\right\rangle,
\end{aligned}
$$

with $\left|\tau_{1}-\tau_{2}\right| \sim\left|\tau_{3}-\tau_{4}\right| \sim d / v_{F} \ll\left|\tau_{1}-\tau_{3}\right| \sim 1 / \Delta$. Also in this case the other relevant processes can be obtained from the one in Fig. 2(b) by means of particle-hole conjugation and by changing the time ordering.

The direct evaluation of averages like (14), (15) with the help of bosonized field operators like (2) is tedious but straightforward. The resulting expressions can be simplified further in the two limiting cases $v_{F} / d \ll \Delta$ and $v_{F} / d \gg \Delta$, which contain all the important physics of the problem.

\section{IV. dC-JOSEPHSON CURRENT THROUGH A QUANTUM WIRE}

We first turn to the geometry depicted in Fig. 1(a). It consists of a quantum wire of length $L \rightarrow \infty$ connected to two superconductors by tunnel junctions separated by a distance $d$. The topological excitations play no role in this case (their energy is vanishingly small) and the wire is described by the nonzero modes only [first term in Eq. (8)].

\section{A. The case $v_{F} / d \ll \Delta$}

The expression for the phase-dependent part of the free energy contains four terms of the type of the first term in Eq. (12) [see Fig. 2(a)]. In this case at low temperatures $T \ll \Delta$ one can approximate the anomalous Green functions (13) by $\delta$ functions in time. This fixes equal time arguments $\tau_{1}=\tau_{2}, \tau_{3}=\tau_{4}$ in $\Pi_{L}^{(a)}$. The remaining integration should be performed over the time $\tau=\tau_{1}-\tau_{3}$. The dominant contribution to the the integral comes from the terms with $n= \pm 1$ in Eq. (2). As a result, the Josephson current (11) through the quantum wire is given by

$$
I_{J}^{(a)}=I_{c}^{(a)}(T) \sin \chi,
$$

with a temperature-dependent critical current

$$
I_{c}^{(a)}(T)=\frac{4 \pi e v_{F}}{d} \frac{G_{1} G_{2}}{\left(4 e^{2}\right)^{2}} F_{w}^{(a)}(T),
$$

where

$$
F_{w}^{(a)}(T)=\left[\frac{1}{k_{F} d}\right]^{2 / g_{\rho}-1} \int_{-\beta v_{F} / 2 d}^{\beta v_{F} / 2 d} \frac{d x}{2 \pi} \prod_{j=\rho, \sigma}\left[\frac{2 \pi^{2} d^{2}}{v_{j}^{2} \beta^{2}} \frac{2}{\cosh \left(2 \pi d / v_{j} \beta\right)-\cos \left(2 \pi d x / \beta v_{F}\right)}\right]^{1 / g_{j}}
$$

(with $g_{\sigma}=2$ and $v_{\sigma}=v_{F}$ ).

In the noninteracting case, at zero temperature, $F_{w}^{(a)}(0)=1$. The Josephson current decreases as $1 / d$ with increasing distance between the tunnel junctions. This is related to the fact that the density of Cooper pairs in the LL decays in space away from each junction. Hence the overlap of the macroscopic wave functions of the two superconductors, which is responsible for the Josephson effect, is suppressed. Repulsive interactions in the wire make the Josephson effect vanish more rapidly with the distance between the superconductors,

$$
I_{c}^{(a)}(0) \propto 1 /\left(k_{F} d\right)^{2 / g_{\rho}}
$$

The electron liquid acquires an additional stiffness against density fluctuations, hence the tunneling between $S$ and LL is suppressed. This fact provides an a posteriori justification of our use of perturbation theory when treating electron tunneling in the presence of repulsive interactions.

We consider now the temperature dependence of the critical current. For noninteracting electrons, $g_{\rho}=2$, the critical current can be calculated explicitly:

$$
\frac{I_{c}^{(a)}(T)}{I_{c}^{(a)}(0)}=\frac{2 \pi T d}{v_{F}} \frac{1}{\sqrt{\cosh ^{2}\left(2 \pi T d / v_{F}\right)-1}} .
$$

At low temperatures, $T \ll v_{F} / 2 \pi d$, the critical current is suppressed below its zero temperature value in a power-law fashion

$$
I_{c}^{(a)}(T) / I_{c}^{(a)}(0) \simeq 1-\frac{2}{3}\left(\frac{\pi T d}{v_{F}}\right)^{2} .
$$

In the high-temperature regime, $T \ll v_{F} / 2 \pi d$, the decay is exponential

$$
\frac{I_{c}^{(a)}(T)}{I_{c}^{(a)}(0)} \simeq \frac{\sqrt{8} \pi T d}{v_{F}} \exp \left(-2 \pi T d / v_{F}\right) .
$$

It is possible to obtain analytical results also in the interacting case. In particular, for weak interaction $2-g_{\rho} \ll 2$ and low temperatures $T \ll v_{F} / 2 \pi d$ the critical current behaves as

$$
\frac{I_{c}^{(a)}(T)}{I_{c}^{(a)}(0)} \simeq 1+\frac{2-g_{\rho}}{4}\left(\frac{2 \pi T d}{v_{F}}\right)^{2 / g_{\rho}}-\frac{2}{3}\left(\frac{\pi T d}{v_{F}}\right)^{2},
$$

where we dropped terms $G\left[\left(2-g_{\rho}\right)^{2}+\left(2-g_{\rho}\right)\left(T d / v_{F}\right)^{2}\right]$. This result can be interpreted in terms of a competition between two effects. At low temperatures the dominant dependence comes from the renormalization of the tunneling amplitudes $T_{1,2}$ in the presence of interaction. ${ }^{14}$ The critical current increases with temperature. Above the crossover temperature $T_{\text {cross }} \simeq(3 / 8)\left(2-g_{\rho}\right) v_{F} / \pi d$ the Josephson current decreases due to the shortening of the phase coherence 


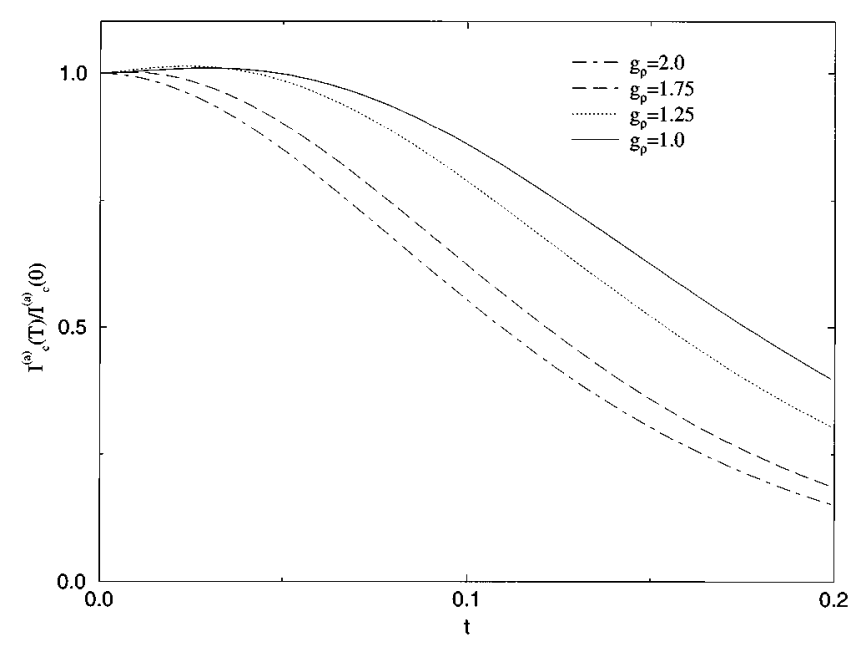

FIG. 3. The critical current of the wire as a function of the temperature $\left(t=T d / 2 \pi v_{F}\right)$ for various values of the interaction strength $g_{\rho}=1.0,1.25,1.75,2.0$.

length. Although the maximum is not very pronounced, the crossover temperature shifts to higher values as the interaction strength increases (see Fig. 3). This results in a wider temperature range in which the critical stays almost constant. It is evident from Eq. (21) that the coefficient responsible for the anomalous temperature dependence vanishes in the absence of interaction, thus restoring the $T^{2}$ suppression of the critical current (20). For high temperatures $T \gg v_{\rho} / 2 \pi d$ the suppression becomes exponential,

$$
I_{c}^{(a)}(T) \propto T^{2 / g_{\rho}} \exp \left(-2 \pi T d / v_{F}\right) .
$$

The full temperature dependence of the critical current, calculated by numerical integration of Eqs. (17), (18) is shown in Fig. 3. We see that for moderate strength of the interaction $g \sim 1$ the Josephson current will maintain an appreciable value up to a temperature $T \sim v_{F} / d$, which is of the order of $0.7 \mathrm{~K}$ for typical experimental parameters $v_{F}=3.0$ $10^{5} \mathrm{~m} / \mathrm{s}$ and $d=3 \mu \mathrm{m} .{ }^{31}$ Moreover, the value of the critical current $I_{c}^{(a)}(T=0) \approx 22 \mathrm{nA}$ [estimated for the parameters given above and $\left.G_{i} /\left(4 e^{2}\right)=0.3\right]$, is large enough to be measured experimentally.
Note that we estimated the Josephson current assuming fixed Josephson phase difference between the superconductors. Thermal fluctuations of the Josephson phase would smear the critical current at temperatures $T^{*} \sim E_{J} \equiv I_{c}^{(a)} / 2 e$, provided that the superconductors are coupled by the LL only. Using Eq. (17) for the noninteracting case [with $\left.F_{w}^{(a)}(0)=1\right]$, one obtains that the temperature $T^{*}$ is by a factor $2 \pi G_{1} G_{2} /\left(4 e^{2}\right)^{2} \ll 1$ smaller than the characteristic temperature scale $v_{F} / d$ for the LL. Hence, in order to observe nontrivial temperature dependence of the critical current, one has to fix the phase difference between the superconductors, e.g., by means of an additional Josephson junction.

\section{B. The case $v_{F} / d \gg \Delta$}

In this limit, the electrons propagate fast and independently through the LL on a time scale $1 / \Delta$. A typical contribution is depicted in Fig. 2(b). The Cooperon (15) can be approximated as

$$
\begin{aligned}
& \Pi_{L}^{(b)}\left(0, d ; \tau_{1}, \ldots, \tau_{4}\right) \\
& \quad \approx\left\langle\hat{\Psi}_{L,+}\left(0, \tau_{1}\right) \hat{\Psi}_{L,+}^{\dagger}\left(d, \tau_{2}\right)\right\rangle\left\langle\hat{\Psi}_{L,-}\left(0, \tau_{3}\right) \hat{\Psi}_{L,-}^{\dagger}\left(d, \tau_{4}\right)\right\rangle,
\end{aligned}
$$

where we substitute

$$
\left\langle\hat{\Psi}_{L,+}\left(0, \tau_{i}\right) \hat{\Psi}_{L,+}^{\dagger}\left(d, \tau_{j}\right)\right\rangle \approx C \delta\left(\tau_{i}-\tau_{j}\right) .
$$

The constant $C$ is determined by integration of the timeordered single-particle correlator of the LL,

$$
C=\int_{-\beta / 2}^{\beta / 2} d \tau\left\langle T_{\tau} \hat{\Psi}_{L,+}(0, \tau) \hat{\Psi}_{L,+}^{\dagger}(d, 0)\right\rangle .
$$

The temperature-dependent Josephson current is found to be

$$
I_{J}^{(b)}=I_{c}^{(b)}(T) \sin \chi,
$$

where the critical current is given by

$$
I_{c}^{(b)}(T)=e \Delta \frac{G_{1} G_{2}}{\left(4 e^{2} / \pi\right)^{2}} F_{w}^{(b)},
$$

with

$$
\begin{aligned}
F_{w}^{(b)}= & {\left[\frac{1}{k_{F} d}\right]^{g_{\rho} / 4+1 / g_{\rho}-1}\left[\frac{g_{\rho}}{2}\right]^{g_{\rho} / 4+1 / g_{\rho}}\left[\frac{\pi d}{\beta v_{F}}\right]^{g_{\rho} / 4+1 / g_{\rho}+1}\left\{\frac{2}{\pi} \int_{0}^{\beta v_{F} / 2 d} d x \sin \left(\frac{\zeta_{\rho}+\zeta_{\sigma}}{2}\right)\right.} \\
& \left.\times \prod_{j=\rho, \sigma}\left[\frac{2}{\cosh \left(2 \pi d / \beta v_{j}\right)-\cos 2 \pi d x / \beta v_{F}}\right]^{g_{j} / 16+1 / 4 g_{j}}\right\}^{2} .
\end{aligned}
$$

The phase factor $\zeta_{j}$ is given by

$$
\zeta_{j}=\arctan \left[\cot \left(\frac{\pi d x}{\beta v_{F}}\right) \tanh \left(\frac{\pi d}{\beta v_{j}}\right)\right] .
$$

In the noninteracting case $g_{\rho}=g_{\sigma}=2, F_{w}^{(b)}$ can be calculated explicitly:

$$
F_{w}^{(b)}=\left[\frac{2}{\pi} \arctan \left(\frac{1}{\sinh \left(\pi d / \beta v_{F}\right)}\right)\right]^{2} .
$$

At zero temperature we find $F_{w}^{(b)}=1$. The resulting Josephson current thus is independent of the distance $d$ between the contacts, analogous to the result obtained in Ref. 6. At finite 
temperatures the Josephson current is suppressed. If $T \ll v_{F} / d$, the suppression is linear in $T$ :

$$
\frac{I_{c}^{(b)}(T)}{I_{c}^{(b)}(0)} \simeq 1-\frac{4 T d}{v_{F}}
$$

In the interacting case, at $T=0$, the critical current is suppressed,

$$
I_{c}^{(b)}(0) \propto 1 /\left(k_{F} d\right)^{g_{\rho} / 4+1 / g_{\rho}-1} .
$$

At finite temperatures, and for weak interactions we obtain

$$
\frac{I_{c}^{(b)}(T)}{I_{c}^{(b)}(0)}-1 \sim-\frac{3}{2 \pi}\left[\frac{\pi T d}{v_{F}}\right]^{1 / 2+g_{\rho} / 8+1 / 2 g_{\rho}}+\frac{2-g_{\rho}}{2} \frac{\pi T d}{v_{F}}
$$

where we dropped terms of the order of $\mathcal{Q}\left[\left(2-g_{\rho}\right)^{2}\right.$ $\left.+\left(2-g_{\rho}\right)\left(T d / v_{F}\right)^{2}\right]$. We find again an anomalous dependence on temperature, like the one we discussed in the case $v_{F} / d \ll \Delta$.

\section{V. dc-JOSEPHSON CURRENT THROUGH A RING}

In case of the ring with circumference $L$ [Fig. 1(b)], we should take into account the contribution to the Josephson current due to the topological part, see Eqs. (4a), (4b), and (8). The Cooperon for the ring in case of a symmetric setup $d=L / 2 \gg v_{F} / \Delta$ is evaluated along the same lines as before. It is then straightforward to get the Josephson current

$$
I_{J}=\frac{2 \pi e v_{F}}{L} \frac{G_{1} G_{2}}{\left(4 e^{2}\right)^{2}} \sum_{\epsilon= \pm 1}\left\langle F_{r}\left(g_{\rho}, L, \epsilon, M_{\sigma}, J_{\rho}\right) \sin \left(\chi+\epsilon \pi M_{\sigma} / 2+\pi J_{\rho} / 2\right)\right\rangle_{J, M},
$$

where

$$
\begin{aligned}
F_{r}= & \frac{1}{2}\left[\frac{\pi}{k_{F} L}\right]^{2 / g_{\rho}-1} \int_{-\pi \beta v_{F} / 2 L}^{\pi \beta v_{F} / 2 L} d x\left[\frac{1}{\cosh \left(2 x / g_{\rho}\right)}\right]^{2 / g_{\rho}} \frac{1}{\cosh (x)} \cosh \left[\left(\frac{2}{g_{\rho}}\right)^{2}\left(M_{\rho}-4 f_{\mu}\right) x+\epsilon J_{\sigma} x\right] \\
& \times \prod_{j=\rho, \sigma}\left[\frac{1+\sum_{n} \exp \left(2 \pi \beta v_{j} n^{2} / L\right)}{1+\sum_{n} \exp \left(2 \pi \beta v_{j} n^{2} / L\right) \cos \left(2 n \lambda_{j}(x)\right)}\right]^{1 / g_{j}}
\end{aligned}
$$

with

$$
\lambda_{j}(x)=\frac{1}{2} \operatorname{arcos}\left[\cosh \left(\frac{2 v_{j} x}{v_{F}}\right)\right]
$$

The brackets, $\langle\cdots\rangle_{J, M}$, should be considered as the thermal average over the topological excitations weighted by the appropriate Boltzmann factor and subject to the topological constraints.

For zero temperature this calculation involves only the ground state (see Ref. 25 for details). The dependence of the critical current on $f_{\Phi}$ and $f_{\mu}$ has been found to show very rich behavior. In the present study, we will focus on the effect of finite temperatures. In particular, we will investigate how robust the structure, found in Ref. 25 is against thermal fluctuations.

Two remarks are in order at this point. First, throughout this section, we assume that the linearization point of the original electron spectrum corresponds to odd values of $N_{0}$ (for even $N_{0}$ the picture is the same, apart from a relative shift of $f_{\Phi}$ and $f_{\mu}$ ). Second, the Josephson current depends periodically on both $f_{\Phi}$ and $f_{\mu}$ with period 1 . However, since the original problem has additional symmetries $f_{\Phi} \rightarrow-f_{\Phi}$ and $f_{\mu} \rightarrow-f_{\mu}$ (together with a change of sign of the corresponding topological numbers, $J_{j}$ and $M_{j}$ ), it is enough to consider $f_{\Phi}$ and $f_{\mu}$ in the intervals $0<f_{\Phi}<1 / 2$ and $0<f_{\mu}<1 / 2$.

It is instructive to discuss first the noninteracting case $g_{\rho}=2$, for $T=0$. If $f_{\mu}+f_{\Phi}<1 / 2$ the ground-state configura- tion of $J_{j}$ and $M_{j}$ is found to be $\left(J_{\rho}, J_{\sigma}, M_{\rho}, M_{\sigma}\right)=$ $(0,0,0,0)$; if $f_{\mu}+f_{\Phi}>1 / 2$ the configuration is $(2,0,2,0)$. Hence, the ground state of the system can be changed by varying either the flux or the gate voltage. As a result, the Josephson current changes, see Eqs. (25), (26). This is illustrated in Fig. 4, where the critical current $I_{c}$ (we write $I_{J}=I_{c} \sin \chi$ where $I_{c}$ can be positive or negative) is plotted as a function of $f_{\mu}$ and $T$ at fixed $f_{\Phi}=0.2$. For $T=0$, the critical current shows a maximum and a sharp jump at $f_{\mu}=0.3$

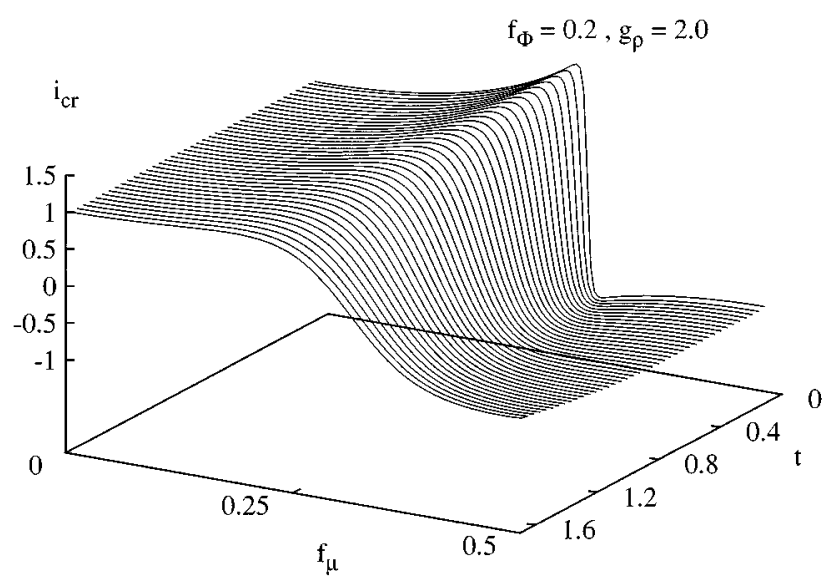

FIG. 4. The critical current through the ring \{normalized to $\left.\left(2 \pi e v_{F} / L\right)\left[G_{1} G_{2} /\left(4 e^{2} / \hbar\right)^{2}\right]\right\}$ at a fixed value of the flux $f_{\Phi}=0.2$ as a function of the gate voltage and the temperature $\left(t=T L / \pi v_{F}\right)$ in the noninteracting case. 
where the states $(0,0,0,0)$ and $(2,0,2,0)$ are degenerate. At this value of the gate voltage, the number of electrons on the ring $\left(M_{\rho}\right)$ increases by two. Since electronic states are doubly degenerate in spin and nonzero flux is applied, the two electrons will occupy the same (clockwise or counterclockwise moving) single-particle state. Therefore, the net current $e v_{F} J_{\rho} / L$ increases by 2 quanta $e v_{F} / L$, while the topological numbers $M_{\sigma}$ and $J_{\sigma}$ related to spin remain unchanged. At the jump, $I_{c}$ changes sign. This reflects the fact that the ring acts as a $\pi$ junction $\left(I_{c}<0\right)$ in the state $(2,0,2,0)$, as can be seen from Eq. (25). Therefore, for noninteracting electrons, the critical current shows two jumps per period of the gate voltage dependence. The same is true for the dependence of $I_{c}$ on the magnetic flux.

This picture is correct for any generic point on the line $f_{\mu}+f_{\Phi}=1 / 2$. At the end points $\left(f_{\mu}, f_{\Phi}\right)=(0,0.5)$ and $(0.5,0)$, no jumps of the critical current occur (one can say that two jumps in opposite directions merge together). Instead, the critical current shows a resonance. The resonance occurs due to alignment of two spin-degenerate energy levels (for clockwise and counterclockwise moving electrons) with the chemical potential of the superconductors. ${ }^{25}$

At a finite temperature, both the nonzero modes and the topological excitations are thermally activated. Thermal activation of the nonzero modes leads to an overall suppression of the critical current, as it has been discussed for the wire in Sec. IV. Thermal activation of the states $(0,0,0,0)$ and $(2,0,2,0)$ will lead to a smearing of the jump. Moreover, at finite temperature there will be a nonvanishing probability to activate other topological excitations which can contribute to the Josephson current. In the plotted temperature range, only one additional state $(1,1,1,1)$ with one extra electron on the ring can be activated. As a result, the negative critical current of the state $(2,0,2,0)$ (at $f_{\mu} \gtrsim 0.3$ ) will be partially compensated by the positive critical current of the state $(1,1,1,1)$, the occupancy of which increases with temperature. Note that the jump at $f_{\mu}=0.3$ remains visible up to temperatures of the order of $T \sim v_{F} / L \sim 1 \mathrm{~K}$ (for the parameters mentioned above and $L=2 \mu \mathrm{m})$. Hence, the parity effect causing the jump is quite robust against thermal fluctuations.

An important feature of the noninteracting case at $T=0$ is that the various possible ground-state configurations may differ by an even number of electrons only. The situation changes drastically when repulsive interactions are switched on. In addition to the states $(0,0,0,0)$ and $(2,0,2,0)$, the state $(1,1,1,1)$ can act as a ground-state configuration. ${ }^{25}$ This happens for parameters $f_{\mu}, f_{\Phi}$ within the range $1+3\left(g_{\rho} / 2\right)^{2}<8\left[f_{\mu}+\left(g_{\rho} / 2\right)^{2} f_{\Phi}\right]<3+\left(g_{\rho} / 2\right)^{2}$. Within this "strip," it is energetically more favorable to add a single electron, rather than a pair of electrons to the ring, due to repulsive electron-electron interactions. The Josephson current in the state $(1,1,1,1)$ differs from the current in the states $(0,0,0,0)$ and $(2,0,2,0)$, see Eqs. (25), (26). For example, for $g_{\rho}=1.75$ and $f_{\Phi}=0.2$ (Fig. 5) the state $(1,1,1,1)$ occurs in the range $0.259<f_{\mu}<0.318$. Indeed, one sees two pronounced jumps of $I_{c}$ at the borders of this interval in Fig. 5 (at low temperatures). Generally, for interacting electrons the critical current shows four jumps per period of the gate voltage dependence. ${ }^{25}$

Similar jumps are seen also at the dependence of the critical current on the flux, Fig. 6. Moreover, $I_{c}$ is a stepwise

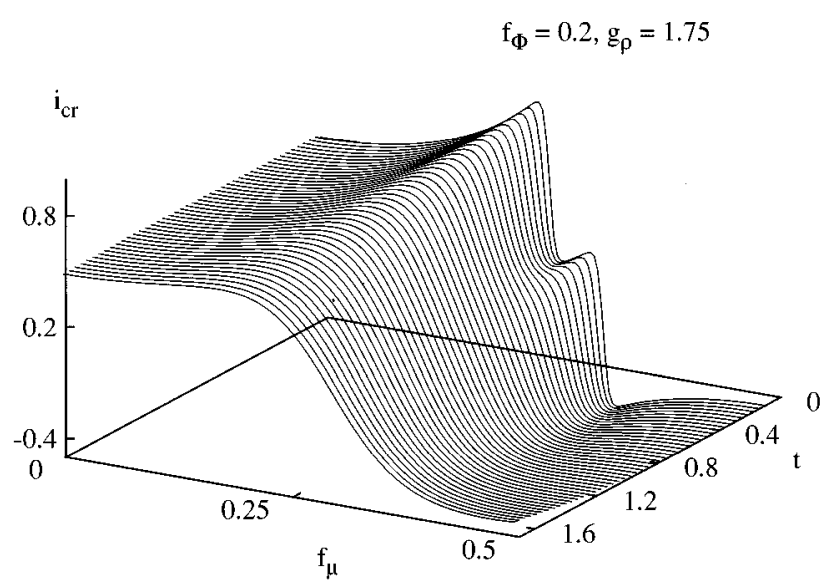

FIG. 5. The same as in the previous figure for the interacting case; for this plot we choose $g_{\rho}=1.75$ but the result is rather generic for repulsive interaction the critical current is normalized to $\left.\left(2 \pi e v_{F} / L\right)\left[G_{1} G_{2} /\left(4 e^{2} / \hbar\right)\right]^{2}\right\}$.

function of $f_{\phi}$ for $T=0$ [this can be seen from Eqs. (25), (26); the flux $f_{\Phi}$ enters to these equations only implicitly, via topological numbers]. Depending on the gate voltage, the critical current can show zero, two, or four jumps per period of the flux dependence. ${ }^{25}$

The state $(1,1,1,1)$ is the ground state in a strip of width $\delta f_{\mu}=\left[1-\left(g_{\rho} / 2\right)^{2}\right] / 4$. This determines the energy $\delta E \simeq\left[1-\left(g_{\rho} / 2\right)^{2}\right] \pi v_{\rho} /\left(g_{\rho} L\right)$ needed to create topological excitations. The features related to the state $(1,1,1,1)$ will be smeared at temperatures $T \sim \delta E$. Therefore, for weak interaction $2-g_{\rho} \ll 1$, the interaction effects will disappear at much lower temperatures $T \ll v_{F} / L$ than the parity effects. For example, the features related to the configuration $(1,1,1,1)$ in Figs. 5 and 6 are seen only in the temperature range $T<\delta E \sim 0.1 \mathrm{~K}$ whereas the overall dependence is robust up to the temperatures $T \sim 1 \mathrm{~K}$. However, it is worthwhile to stress that the $(1,1,1,1)$ state survives at much higher temperatures when the interaction strength is increased.

The behavior we described here is rather generic for all values of $f_{\mu}, f_{\Phi}$ and for various values of the interaction.

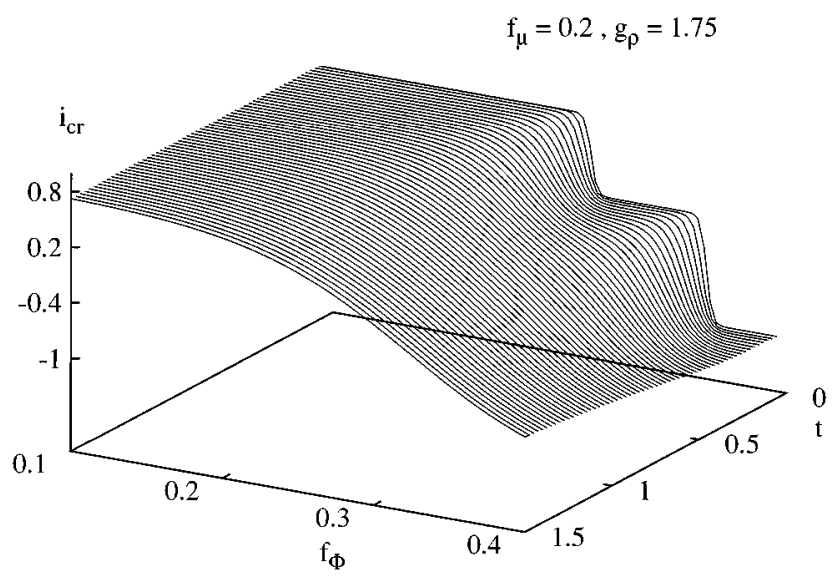

FIG. 6. The critical current through the ring at a fixed value of the gate voltage $f_{\mu}=0.2$ as a function of the flux and the temperature $\left(t=T L / \pi v_{F}\right)$ in the interacting case $g_{\rho}=1.75$ the critical current is normalized to $\left.\left(2 \pi e v_{F} / L\right)\left[G_{1} G_{2} /\left(4 e^{2} / \hbar\right)^{2}\right]\right\}$. 


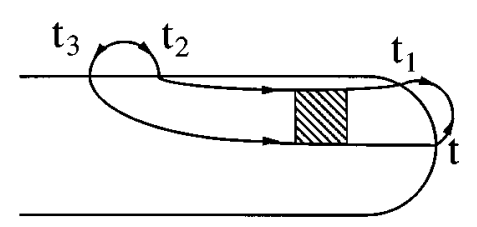

(a)

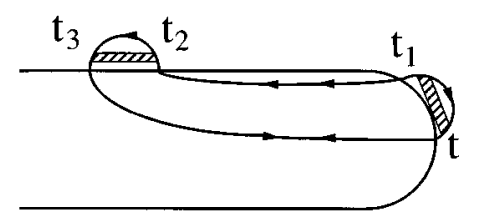

(b)

FIG. 7. Relevant diagrams for ac-Josephson current: (a) $v_{F} / d \gg \Delta$ and (b) $v_{F} / d \ll \Delta$. The shaded area indicates the electronelectron interaction.

What is specific is the configuration of the two superconductors: they are connected symmetrically to the ring. If the points on the ring at which the electrodes are attached would form a generic angle, a more complicated interference pattern would arise. In the symmetric setup, the maximum Josephson current occurs either at $\chi=0$ or at $\chi=\pi$. In the nonsymmetric setup the maximum Josephson current would occur at a value $\chi\left(J_{\rho}, J_{\sigma}, M_{\rho}, M_{\sigma}\right)$ which depends on the values of topological numbers. The critical current should then be found by maximizing the resulting function of the phase difference.

\section{VI. ac-JOSEPHSON EFFECT}

The effect of a finite dc bias voltage $e V \ll 2 \Delta$ applied between the superconductors $S 1$ and $S 2$, will be twofold. First of all, the phase difference $\chi$ between $S 1$ and $S 2$ will acquire a time dependence, according to the Josephson relation $\dot{\chi}=\omega_{J}=2 \mathrm{eV}$. As a result, the Josephson current will oscillate as a function of time at a frequency $\omega_{J}$ (acJosephson effect, see Ref. 32). Secondly, a dc subgap current will be induced, due to Andreev reflection at both junctions. This current is dissipative, energy will be dissipated in the LL. In a typical experiment one thus will find a current with both a dc and an ac component. In this section, we will mainly concentrate on the ac-Josephson current, and estimate the dc component at the end.

In the presence of a bias voltage $V$ between the superconductors, the imaginary time formalism cannot be applied and Josephson current is found by calculating the average of the corresponding Heisenberg operator. Using the interaction representation with the unperturbed Hamiltonian $\hat{H}_{0}$, see Eq. (9), one obtains

$$
\begin{gathered}
I(t)=\left\langle\hat{U}^{\dagger}(t) \hat{I}(t) \hat{U}(t)\right\rangle, \\
\hat{U}(t)=T \exp \left[-i \int_{-\infty}^{t} \hat{H}_{T}\left(t^{\prime}\right) d t^{\prime}\right] .
\end{gathered}
$$

We expand (27) to the fourth order in $\hat{H}_{T}$ and keep the Josephson terms in the current. These are proportional to $\exp ( \pm 2 i e V t)$. As a result the Josephson current is given by an expression which has the same structure as Eq. (12). The integrals are now taken over real times. It is convenient to depict the times $t, t_{1}, t_{2}, t_{3}$ of tunneling events on the Keldysh contour $^{33}$ (the Josephson current is calculated at a time $t)$. Again, we will consider two cases of long $\left(v_{F} / d \ll \Delta\right)$ and short $\left(v_{F} / d \gg \Delta\right)$ distance between the contacts. The relevant diagrams are shown in Fig. 7 for both cases. We restrict our consideration to the case of a quantum wire at zero temperature.

\section{A. The case $v_{F} / d \ll \Delta$}

For a large distance between the contacts the tunneling of two electrons to/from a superconductor is a fast process on the time scale of their propagation through LL. The Josephson current is described by diagrams of the type shown in Fig. 7(a). The Josephson current is then given by

$$
\begin{aligned}
I_{J}(t)= & 4 \pi^{2} e v_{F}^{2} \frac{G_{1} G_{2}}{\left(4 e^{2}\right)^{2}} \\
& \times \operatorname{Re}\left[\sum_{ \pm} \pm e^{ \pm 2 i e V t} \int_{0}^{\infty} d t^{\prime} e^{\mp i e V t^{\prime}} \Pi\left(t^{\prime}\right)\right],
\end{aligned}
$$

where $\Pi(t)=\Pi_{L}^{(a)}(0, d ; i t, i t, 0,0)$ is the Cooperon propagator (14) in real time taken at coinciding time arguments. The leading contribution stems from the terms in Eq. (2) with $n= \pm 1$,

$\Pi(t)=2 \rho_{0}^{2} \prod_{j=\rho, \sigma}\left\{\left[1+i k_{F}\left(v_{j} t+d\right)\right]\left[1+i k_{F}\left(v_{j} t-d\right)\right]\right\}^{-1 / g_{j}}$.

In particular, for noninteracting electrons $\left(g_{\rho}=2\right)$ we obtain

$$
I_{J}(t)=\frac{2 \pi e v_{F}}{d} \frac{G_{1} G_{2}}{\left(4 e^{2}\right)^{2}} \sin \left(2 e V t-\frac{e V d}{v_{F}}\right) .
$$

This result means that the Josephson current acquires an additional phase shift due to the propagation of electrons between the contacts. For interacting electrons we computed the Josephson current numerically. We split $I_{J}$ into sinusoidal and cosinusoidal components,

$$
\begin{aligned}
I_{J}(t)= & \frac{2 \pi e v_{F}}{d} \frac{G_{1} G_{2}}{\left(4 e^{2}\right)^{2}}\left(\frac{1}{k_{F} d}\right)^{2 / g_{\rho}-1}\left\{J_{s}\left(\frac{e V}{v_{F} / d}, g_{\rho}\right) \sin (2 e V t)\right. \\
& \left.+J_{c}\left(\frac{e V}{v_{F} / d}, g_{\rho}\right) \cos (2 e V t)\right\} .
\end{aligned}
$$

The amplitudes $J_{c(s)}$ of the two components and the phase $\varphi=-\arctan \left(J_{c} / J_{s}\right)$ of the Josephson current are shown in Fig. 8 as functions of the voltage for two values of the interaction parameter, $g_{\rho}=1.75$ and 1 . One sees that the deviation from the simple result (30) for noninteracting electrons [which corresponds to $J_{s}=\cos \left(e V d / v_{F}\right), J_{c}=-\sin \left(e V d / v_{F}\right)$, and $\left.\varphi=e V d / v_{F}\right]$ increases with the increase of the interaction. This deviation becomes striking in the dependence of the ac current amplitude $J_{a}=\sqrt{J_{s}^{2}+J_{c}^{2}}$ on the voltage, Fig. 9 . Apart from the noninteracting case $\left[J_{a}(V)=\right.$ const $]$, one sees pronounced oscillations of the current amplitude. These oscillations are due to the difference in the velocities of the charge $\left(v_{\rho}\right)$ and spin $\left(v_{\sigma}\right)$ excitations. The period $\delta V$ of the 


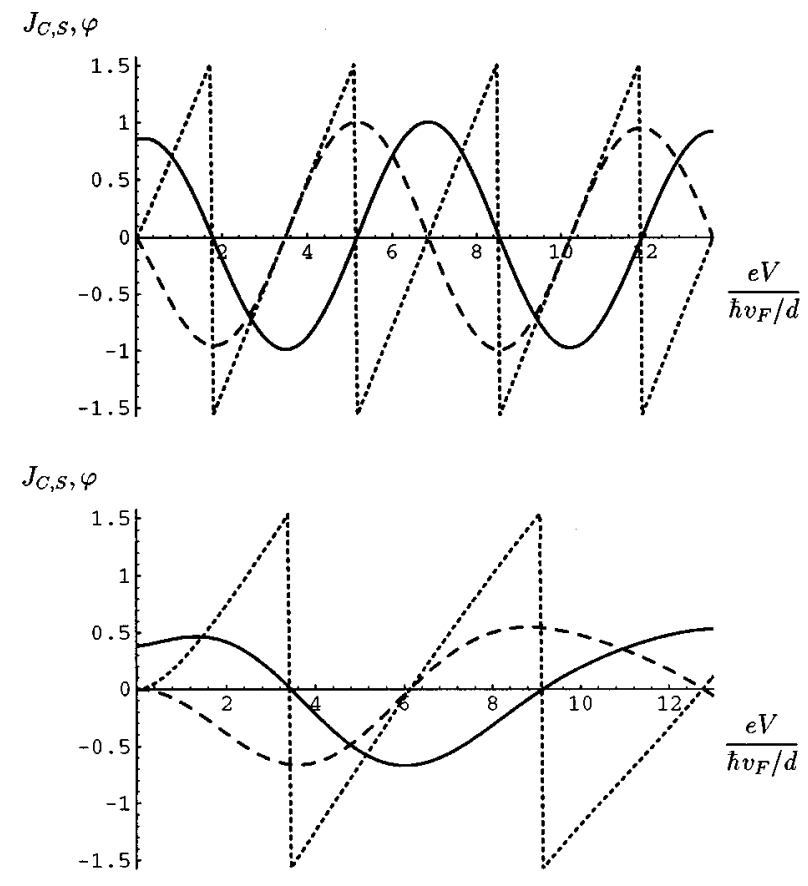

FIG. 8. The voltage dependence of sinusoidal (solid line) and cosinusoidal (dashed line) components and the phase (dotted line) of ac-Josephson current; (a) $g_{\rho}=1.75$ and (b) $g_{\rho}=1$.

oscillations corresponds to $2 \pi$ difference between the phases of charge $\left(e V d / v_{\rho}\right)$ and spin $\left(e V d / v_{F}\right)$ excitations. Using the relation $v_{\rho}=2 v_{F} / g_{\rho}$ we obtain $e \delta V /\left(v_{F} / d\right)$ $=2 \pi\left(1-g_{\rho} / 2\right)^{-1} \simeq 50.4,12.6$ for $g_{\rho}=1.75$ and 1 , respectively. This is in very good argeement with the period of oscillations in Fig. 9. Therefore, the ac-Josephson effect can be used as a tool for the observation of spin-charge separation in the LL.

\section{B. The case $v_{F} / d \gg \Delta$}

At short distances between the contacts, the two electrons propagate fast through the LL on the time scale $1 / \Delta$. The relevant diagrams are similar to the graph shown in Fig. 7(b). The main contribution to the Josephson current comes from the integration of the two-particle propagators of the type $\Pi_{L}^{(b)}\left(0, d ; i t, i t_{1}, i t_{2}, i t_{3}\right)$, Eq. (15) (with possible permuta-

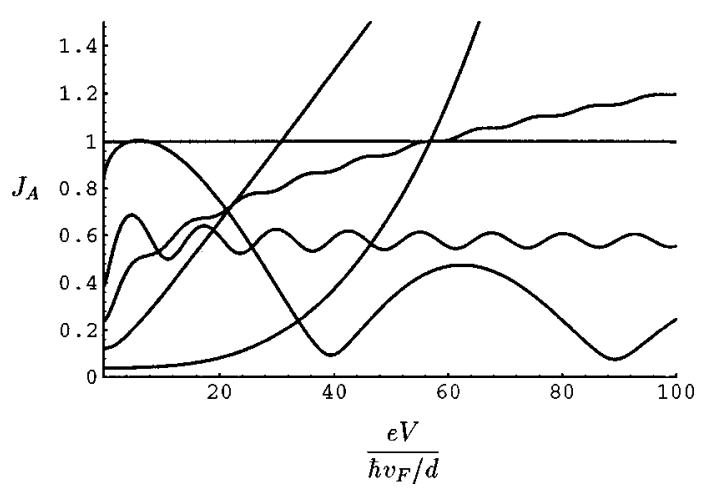

FIG. 9. The voltage dependence of the amplitude of acJosephson current. Here, $g_{\rho}=2,1.75,1,0.75,0.5,0.25$ for the curves from top to bottom at zero voltage. tions of creation and annihilation operators) over the range $t-t_{1} \sim t_{2}-t_{3} \sim d / v_{F}$ and $t-t_{2} \sim 1 / \Delta$ [see Fig. 7(b)]. For this reason, we can present $\Pi_{L}^{(b)}$ as a product of two singleparticle propagators and integrate the latter over the "fast" variables $t-t_{1}$ and $t_{2}-t_{3}$ (from 0 to $\infty$ ) as we did for the dc case. The last integration over the "slow" variable $s=t-t_{2}$ involves the product of two anomalous Green functions with an exponent containing the time-dependent Josephson phase:

$$
\int_{0}^{\infty} d s F_{S 1}^{+}(0, i s) F_{S 2}(0, i s) \exp (i e V s) .
$$

Hence, for short distances between the contacts the presence of LL does not influence the voltage dependence of acJosephson current. The latter is still given by the simple formula

$$
I_{J}(t)=(2 / \pi) K(e V / 2 \Delta) I_{c}^{(b)}(0) \sin (2 e V t)
$$

where $K(x)$ is an elliptic integral and $I_{c}^{(b)}(0)$ is the critical current in the dc case [cf. Eq. (23) in the limit of zero temperature]. The effect of the interaction is only to reduce the value of the critical current, while its voltage dependence is analogous to that of the critical current of a conventional Josephson junction. ${ }^{34}$

We conclude this section with an estimate of the dissipative dc current due to Andreev reflection at both junctions. For a single junction between a superconductor and a LL with repulsive interactions, the subgap current $I_{s}(V)$ as a function of the applied voltage $V$ is given by ${ }^{26,27,35}$ $I_{s}(V) \sim V|V|^{2 / g_{\rho}-1}$. For the system of Fig. 1(a), which consists of two junctions in series, the lowest-order contribution to the subgap current stems from sequential tunneling. Employing a rate equation approach, we find for this contribution

$$
\begin{aligned}
I_{s}(V)= & 2 \pi e \frac{G_{1} G_{2}}{\left(4 e^{2}\right)^{2}} 2 e V\left[\frac{2 e V}{v_{F} k_{F}}\right]^{2 / g_{\rho}-1} \frac{\left(2 / g_{\rho}\right)^{2 / g_{\rho}}}{\Gamma\left(1+2 / g_{\rho}\right)} \\
& \times\left[\frac{2\left(G_{1} G_{2}\right)^{g_{\rho} / 2}}{\left(G_{1}^{2}\right)^{g_{\rho} / 2}+\left(G_{2}^{2}\right)^{g_{\rho} / 2}}\right]^{2 / g_{\rho}} .
\end{aligned}
$$

Comparing this result with the critical current we see that the dissipative component is much smaller at low voltages. In order to get a complete description at finite voltages, one has to solve the corresponding equation for nonlinear resistively shunted-junction model. ${ }^{9}$ This will be discussed in a forthcoming publication. ${ }^{36}$

\section{DISCUSSION}

In this paper, we studied the ac- and dc-Josephson effect in a single-mode quantum wire and quantum ring connected to two superconductors by tunnel junctions. Repulsive interactions were treated in the framework of the Luttinger model. Interactions were found to have a drastic influence on both dc- and ac-Josephson effect.

The critical current is suppressed by interactions at zero temperature. The results depend on the ratio between the characteristic energy $\hbar v_{F} / d$ of the 1D electron system and the superconducting energy gap $\Delta$. For large distances between the contacts $d \gg \hbar v_{F} / \Delta$ in the presence of interactions, 
there is a competition between thermal suppression of coherent two-particle propagation in the wire and activation of tunneling at the junctions at low temperatures. As a result, the critical current shows maximum as a function of temperature. At even higher temperatures, $k_{B} T \gg \hbar v_{\rho} / 2 \pi d$, the suppression becomes exponential.

In our model it is assumed that the superconducting electrodes do not influence the uniformity of the potential along the quantum wire, since they are separated from the wire by thick barriers. It was argued in Ref. 37 that a nonuniform potential in the wire will lead to an effective change of the boundary conditions for the electronic wave function, which in turn could strongly affect our results. However, a recent calculation $^{26}$ of the Josephson current through an interacting quantum wire of finite length is in agreement with our results. This indicates that the results obtained are robust with respect to the specific choice of boundary conditions. They rather describe generic properties of the superconductorLuttinger-liquid system.

If a finite voltage $V$ is applied between the junctions, the ac-Josephson effect occurs. The ac current acquires phase shift proportional to the distance between the tunnel junctions. Moreover, the amplitude of ac current depends on the voltage in an oscillatory fashion due to spin-charge separa- tion. The corresponding period depends on the ratio of the velocities of the spin and charge excitations in the LL.

A quantum wire closed to a loop (or quantum ring) shows interesting parity effects. Boundary conditions on the electronic wave functions result in a discrete set of quantum numbers, related to the number of particles and angular momentum. We showed how these numbers can be tuned by applying a gate voltage and a magnetic flux, and calculated the corresponding dependence of the critical current on these parameters. This dependence shows a rich behavior, which can be detected in an interference experiment employing a superconducting quantum interference device. We showed that the dependence is robust to thermal fluctuations up to experimentally measurable temperatures.

\section{ACKNOWLEDGMENTS}

We would like to thank I.L. Aleiner, L.I. Glazman, Yu.V. Nazarov, G. Schön, C. Winkelholz, and A.D. Zaikin for useful discussions. The financial support of the European Community (HCM-network CHRX-CT93-0136 and HCM ERBCHBI-CT94-1474), the Deutsche Forschungsgemeinschaft through SFB 195, and the Netherlands Organization for Scientific Research (NWO) is gratefully acknowledged. The authors also acknowledge the kind hospitality of ISI-Torino (Italy) where part of this work was done.
*Permanent address.

${ }^{1}$ J. Nitta, T. Azaki, H. Takayanagi, and K. Arai, Phys. Rev. B 46, 14286 (1992); A. Dimoulas, J.P. Heida, B.J. van Wees, T.M. Klapwijk, W.v.d. Graaf, and G. Borghs, Phys. Rev. Lett. 74, 602 (1995).

${ }^{2}$ A. Kastalsky, A.W. Kleinsasser, L.H. Greene, R. Bhat, F.P. Milliken, and J.P. Harbison, Phys. Rev. Lett. 67, 3026 (1991).

${ }^{3}$ A.F. Andreev, Zh. Éksp. Teor. Fiz. 46, 1823 (1964) [Sov. Phys. JETP 19, 1228 (1964)].

${ }^{4}$ For a review, see, C.W.J. Beenakker, in Mesoscopic Quantum Physics, edited by E. Akkermans, G. Montambaux, and J.-L Pichard (North-Holland, Amsterdam, 1995), and references therein.

${ }^{5}$ Several papers, in Mesoscopic Superconductivity, edited by F.W.J. Hekking, G. Schön, and D.V. Averin, Physica B 203, 205 (1994) deal with superconductor-semiconductor devices.

${ }^{6}$ C.W.J. Beenakker and H. van Houten, Phys. Rev. Lett. 66, 3056 (1991); A. Furusaki, H. Takayanagi, and M. Tsukada, ibid. 67, 132 (1991).

${ }^{7}$ B.J. van Wees, P. de Vries, P. Magneé, and T.M. Klapwijk, Phys. Rev. Lett. 69, 510 (1992).

${ }^{8}$ F.W.J. Hekking and Yu.V. Nazarov, Phys. Rev. Lett. 71, 1625 (1993); Phys. Rev. B 49, 6847 (1994).

${ }^{9}$ L.G. Aslamazov, A.I. Larkin, and Yu.N. Ovchinnikov, Zh. Éksp. Teor. Fiz. 55, 323 (1968) [Sov. Phys. JETP 28, 171 (1969)].

${ }^{10}$ For a review, see, Single Charge Tunneling, edited by H. Grabert and M.H. Devoret (Plenum, New York, 1992).

${ }^{11}$ R. Bauernschmitt, J. Siewert, Yu.V. Nazarov, and A.A. Odintsov, Phys. Rev. B 49, 4076 (1994).

${ }^{12}$ B.L. Altshuler, D.E. Khmelnitskii, and B.Z. Spivak, Solid State Commun. 48, 841 (1983).

${ }^{13}$ For a review, see, V.J. Emery, in Highly Conducting OneDimensional Solids, edited by J.T. Devreese, R.P. Evrard, and V.E. van Doren (Plenum, New York, 1979); J. Sólyom, Adv.
Phys. 28, 201 (1979); D. Jerome and H.J. Schulz, ibid. 31, 299 (1992); J. Voit, Rep. Prog. Phys. 58, 977 (1995).

${ }^{14}$ C.L. Kane and M.P.A. Fisher, Phys. Rev. Lett. 68, 1220 (1992); Phys. Rev. B 46, 15233 (1992).

${ }^{15}$ K.A. Matveev and L.I. Glazman, Phys. Rev. Lett. 70, 990 (1993); K.A. Matveev, D. Yue, and L.I. Glazman, ibid. 71, 3351 (1993).

${ }^{16}$ P. Fendley, A.W.W. Ludwig, and H. Saleur, Phys. Rev. Lett. 74, 3005 (1995).

${ }^{17}$ U. Weiss, R. Egger, and M. Sassetti, Phys. Rev. B 52, 16707 (1995).

${ }^{18}$ D. Loss, Phys. Rev. Lett. 69, 343 (1992).

${ }^{19}$ These parity effects, occurring in a normal system, are quite different from the ones discussed in Ref. 11 which are related to superconductivity.

${ }^{20}$ F.D.M. Haldane, Phys. Rev. Lett. 47, 1840 (1981); J. Phys. C 14, 2585 (1981).

${ }^{21}$ N. Byers and C.N. Yang, Phys. Rev. Lett. 7, 46 (1961).

${ }^{22}$ D. Loss and P. Goldbart, Phys. Rev. B 43, 13762 (1991); S. Fujimoto and N. Kawakami, ibid. 48, 17406 (1993).

${ }^{23}$ A.R. Goñi, A. Pinczuk, J.S. Weiner, J.M. Calleja, B.S. Dennis, L.N. Pfeiffer, and K.W. West, Phys. Rev. Lett. 67, 3298 (1991).

${ }^{24}$ S. Tarucha, T. Honda, and T. Saku, Solid State Commun. 94, 413 (1995).

${ }^{25}$ A.A. Odintsov, R. Fazio, and F.W.J. Hekking, in Mesoscopic Superconductivity (Ref. 5), p. 361; R. Fazio, F.W.J. Hekking, and A.A. Odintsov, Phys. Rev. Lett. 74, 1843 (1995).

${ }^{26}$ D.L. Maslov, M. Stone, P.M. Goldbart, and D. Loss (unpublished).

${ }^{27}$ M.P.A. Fisher, Phys. Rev. B 49, 14550 (1994).

${ }^{28}$ The tunnel junctions are assumed to have linear dimensions that are small compared to their separation $d$, but large compared to the Fermi wavelength $\lambda_{F}$.

${ }^{29}$ A.A Abrikosov, L.P. Gorkov, and I.E. Dzyaloshinski, Methods of 
Quantum Field Theory in Statistical Physics (Dover, New York, 1975).

${ }^{30}$ For BCS superconductors in the clean limit, the gap $\Delta$ is given by $v_{F, S} / \xi$, where $\xi$ is the correlation length. Therefore, comparing energy scales means simply comparing $d$ and $\xi$, up to the ratio $v_{F} / v_{F, S}$ of Fermi velocities in the $1 \mathrm{D}$ system and the superconductor.

${ }^{31}$ D. Mailly, C. Chapelier, and A. Benoit, Phys. Rev. Lett. 70, 2020 (1993).

${ }^{32}$ M. Tinkham, Introduction to Superconductivity (Krieger, Mala- bar, FL, 1980).

${ }^{33}$ L.V. Keldysh, Zh. Éksp. Teor. Fiz. 47, 1515 (1964) [Sov. Phys. JETP 20, 1018 (1965)].

${ }^{34}$ I.O. Kulik and I.K. Yanson, The Josephson Effect in Superconducting Tunneling Structures (Israel Program for Scientific Translations, Jerusalem, 1972).

${ }^{35}$ C. Winkelholz, R. Fazio, F.W.J. Hekking, and G. Schön (unpublished).

${ }^{36}$ R. Fazio, F.W.J. Hekking, and A.A. Odintsov (unpublished).

${ }^{37}$ M. Fabrizio and A. Gogolin, Phys. Rev. B 51, 17827 (1995). 